\title{
Normativas EN-9100 e ISO-9000 en el sector aeroespacial español
}

\author{
Castillo Peces, Carlos* \\ Mercado Idoeta, Carmelo** \\ Prado Román, Camilo*** \\ Soto González, Federico ${ }^{\star \star \star \star}$
}

\section{Resumen}

El sector aeroespacial es uno de los más importantes tanto a nivel mundial como en España y se caracteriza por un alto nivel de implantación de Normas de Aseguramiento de la Calidad. La Normativa EN 9100 supone la adaptación de las Normas ISO 9100 a las especificidades de dicho sector. El objetivo del presente trabajo, es analizar los resultados obtenidos al implantar la Normativa EN 9100, y compararlos con los que se derivan de la adhesión a las Normas ISO 9000, en las empresas del sector aeroespacial español. Para ello se envió un cuestionario a los directores de calidad de las 355 empresas que configuran este sector, recibiéndose 115 cuestionarios válidos, cuyas respuestas fueron tratadas mediante un análisis descriptivo y ANOVA. Los resultados muestran que en el sector aeroespacial español, la implantación de la norma EN 9100 promueve mayor impacto positivo que la norma ISO 9000 en todos los aspectos considerados, tanto "internos" (procesos organizativos, ejecución de operaciones, y gestión de recursos humanos), como "externos"(financiero-comerciales, y de relación con los clientes). Destacan especialmente, de los efectos positivos "internos," los relativos a los procesos organizativos, y de los efectos positivos "externos" la gestión de la "relación con la clientela".

Palabras clave: Gestión de calidad, normas aseguramiento de la calidad, Normativa EN 9100, Normativa ISO 9000, sector aeroespacial español.

\section{Recibido: 18-04-1.3 Aceptado: 01-06-14}

* Doctor en Ciencias Económicas y Empresariales por la Universidad Rey Juan Carlos. Profesor Titular del Departamento de Economía de la Empresa y Director del Master Oficial de Marketing de la citada Universidad. E-mail: carlos.delcastillo@urjc.es

** Doctor en Ciencias Económicas y Empresariales por la Universidad Complutense de Madrid. Profesor Titular de Comercialización e Investigación de Mercados de la Universidad Rey Juan Carlos, y Director del Departamento de Economía de la Empresa de la URJC. E-mail: carmelo.mercado@urjc.es

*** Doctor en Ciencias Económicas y Empresariales por la Universidad Rey Juan Carlos. Profesor Titular de Finanzas de la Universidad Rey Juan Carlos. Especialista en Investigación Cuantitativa y Técnicas Estadísticas por la Universidad Politécnica de Madrid. E-mail: camilo.prado.roman@urjc.es.

**** Doctor en Ciencias Económicas y Empresariales por la Universidad Rey Juan Carlos. Consultor de Estrategia en la Dirección de Estrategia Corporativa en IBERIA Líneas Aéreas de España. E-mail: f.soto@iberia.es 


\section{Standards EN-9100 and ISO-9000 in the Spanish Aerospace Sector}

The aerospace sector is one of the most important on a world level as well as in Spain, and it is characterized by a high level of implantation of quality assurance standards. Standard EN 9100 supposes the adaptation of the ISO 9100 Standards to the specifics of the aforementioned sector. The objective of this work is to analyze the results obtained by implanting the EN 9100 Standards and compare them with those derived from adherence to the ISO 9000 Standards in Spanish aerospace sector companies. To accomplish this, a questionnaire was sent to quality directors at the 355 companies that make up this sector. One-hundred fifteen valid questionnaires were received, whose responses were treated using descriptive analysis and ANOVA. Results show that in the Spanish aerospace sector, implantation of the EN 9100 standard promotes a greater positive impact than the ISO 9000 standards in all aspects considered, both "internal" (organizational processes, execution of operations and human resource management), as well as "external" (financing-commercial and client relations). Among the positive internal effects, those related to organizational processes stand out especially, and for the positive external effects, client relations management is cited.

Key words: Quality management, quality assurance standards, EN 9100 Standards, ISO 9000 Standards, Spanish aerospace sector.

\section{Introducción}

La industria aeroespacial es uno de los sectores más relevantes de la economía, de tal forma que la facturación de las 100 primeras empresas a nivel mundial, ha sido en 2011 de 665.970 millones de dólares, lo que supone aproximadamente un $5 \%$ del producto interior bruto mundial (PwC, 2012). Así mismo, la actividad de este sector se desarrolla en un entorno cada vez más competitivo, debido a la presión de los clientes (aerolíneas y propietarios de aeronaves principalmente) y de los propios fabricantes de equipos. Por otro lado, la progresiva complejidad de los sistemas utilizados en este sector, así como la importancia de los proyectos a acometer, han provocado que en la actualidad no exista ningún fabricante de producto final (principalmente aeroplanos, helicópteros y satélites) que desarrolle en su totalidad el producto, pues éste se obtiene como resultado de la colaboración de gran cantidad de empresas especialistas en sistemas, subsistemas y otros componentes específicos de dicho producto final, lo que origina unas relaciones de subcontratación entre todas las empresas participantes en la cadena de valor muy notables ${ }^{1}$, así como una progresiva internacionalización de las actividades de fabricación y desarrollo. Un ejemplo de lo comentado lo constituye el 
consorcio europeo Airbus, cuyos productos finales implican la colaboración de al menos cinco de los países que lo componen (Francia, España, Alemania, Reino Unido y Rusia), además de participar en cada filial de cada país diversos proveedores y empresas auxiliares.

El sector aeroespacial español presenta unas características similares a las descritas para este sector a nivel mundial, por su nivel de competencia y complejidad, y también por su relevancia, ya que según datos correspondientes al año 2011 (DBK, 2012), se trata de la quinta industria europea del sector, tanto por nivel de empleo (40.200 empleados) como por volumen de facturación (6.715 millones de euros), con una clara vocación exportadora, que alcanza a casi el $75 \%$ del total facturado en el año.

La complejidad del sector, junto con el hecho de que la "seguridad" sea el aspecto central que sustenta su actividad, provoca que los productos aeroespaciales estén sometidos a muy altas exigencias en temas de calidad, fiabilidad y mantenibilidad, de tal forma que todos los productos estén definidos basándose en unos estándares de calidad elevados y comunes para todas las empresas que lo conforman, buscando que dichos productos sean al mismo tiempo seguros y competitivos. Esto provoca que la normativa de calidad en el sector sea una de las más exigentes en el ámbito industrial (Grijalbo y Prida, 2005a).

Para lograr estos elevados estándares de la calidad, las empresas de este sector han venido aplicando diversos sistemas de gestión de la calidad, que se estudiarán con más detalle en el siguiente apartado de este trabajo, dentro de los cuales los más extendidos son las Normas de Aseguramiento de la Calidad ISO 9000 , y sobre todo la serie de Normas EN 9100 , que supone especificidades propias de esta industria respecto a ISO 9000, que es de índole más genérica.

Estas normas de aseguramiento de la calidad tienen como base la sistematización y formalización de tareas para lograr una uniformidad en el producto o servicio, así como la conformidad en el cumplimiento de las especificaciones establecidas por el cliente (Anderson et al., 1999), es decir, estandarizan procedimientos, funciones y roles, y no tanto el logro de objetivos o resultados (Guler et al., 2002; Braun, 2005). Por otro lado, la estandarización es un mecanismo que favorece el intercambio y el comercio internacional propios de una economía global como la actual, al eliminar los obstáculos derivados de las prácticas de cada país (Heras y Boiral, 2013), por lo que da respuestas al carácter internacional de los proyectos de colaboración entre empresas de distintos países que son habituales en esta industria.

La implantación de estas Normas de Aseguramiento de la Calidad, lleva aparejados una serie de costos iniciales y de mantenimiento, que en el caso de EN 9100 serán superiores a los de la Normativa genérica ISO 9000, al incluir una serie de requisitos adicionales propios del sector aeroespacial, que en teoría deberían compensarse con los beneficios obtenidos de dichas Normas. Sin embargo, según investigaciones previas realizadas sobre ISO 9000, los resultados derivados de su implantación no siempre compensan dichos costos (entre otros Terziosvski et al., 1997; Gavin, 2000; Singles et 
al., 2001; Wayhan et. al., 2002; Martínez-Costa y Martínez-Lorente, 2007).

La revisión bibliográfica realizada demuestra que existen pocos trabajos relacionados con la Normativa EN $9100^{2}$, y éstos se focalizan fundamentalmente en las características diferenciales de esta Norma respecto a su predecesora (IS0 9000), así como en la forma de llevar a cabo su implantación en algunas de las principales empresas de este sector (EADS-CASA, SENER, ITP, entre otras), pero ninguno de ellos hace referencia a los resultados derivados de su implantación. Por otro lado, en lo referente a la familia de Normas ISO 9000, aunque existen múltiples estudios sobre los beneficios producidos por la adhesión a las mismas, ninguno de ellos se refiere al sector aeroespacial.

La escasez de trabajos sobre los resultados de la aplicación de las Normas de Aseguramiento de la Calidad en el sector aeroespacial, así como la importancia del mismo, han impulsado esta investigación, cuyo objetivo lo constituye el análisis de los resultados derivados de la implantación de la Normativa EN 9100 en comparación con los generados con la adhesión a ISO 9000, en las empresas del sector aeroespacial español. Para ello, previamente se analizarán de forma individual los posibles beneficios que cada una de estas Normativas han producido en dichas empresas, para con posterioridad proceder al análisis comparativo de los resultados obtenidos, y comprobar de esta manera si al implantar EN 9100 se generan unos beneficios diferenciales que puedan justificar los costos adicionales de implantación de dicha Normativa.

La investigación se dirigió a las empresas del sector aeroespacial español, es decir, a todas aquellas que participan de manera directa en el desarrollo, fabricación y/o mantenimiento de productos aeroespaciales. Para la fijación del censo a investigar, se descartó como fuente la Clasificación Nacional de Actividades Económicas (CNAE), que es el principal censo de empresas españolas, clasificadas en base a su sector de actividad, puesto que no existe una o varias categorías que recojan a todas las empresas del sector. Esto es debido a que este sector está compuesto por una gran diversidad de empresas, tanto a nivel de tamaño como de especialización empresarial.

El análisis de los estudios previos sobre los resultados de la implantación de las Normas de Aseguramiento de la Calidad, cuyos autores y conclusiones más relevantes se detallan en la sección 3 de este manuscrito, muestra que existen pocos trabajos relacionados con la Normativa EN 9100, y éstos se focalizan fundamentalmente en las características diferenciales de esta Norma respecto a su predecesora (IS0 9000), así como en la forma de llevar a cabo su implantación en algunas de las principales empresas de este sector, como son, entre otras, Construcciones Aeronáuticas S.A. (EADS-CASA), SENER Ingeniería y Sistemas S.A., e Industria de Turbopropulsores S.A. (ITP); sin embargo, ninguno de ellos hace referencia a los resultados derivados de su implantación. Por otro lado, en lo referente a la familia de Normas ISO 9000 , aunque existen múltiples estudios sobre los beneficios producidos por la adhesión a las mismas, ninguno de ellos se refiere al sector aeroespacial. 
Por este motivo se ha tenido que recurrir a la utilización de una compilación de bases de datos específicas del sector que aglutinan a sus empresas, entre las que destacan el censo de la Asociación Española de Constructores de Material Aeroespacial (ATECMA), que al cierre de 2008 era la asociación de referencia en España (sus 55 empresas asociadas aglutinaba al $90 \%$ de su facturación), así como la base de datos OASIS, que registra todas las empresas del sector aeroespacial adheridas a EN 9100. En definitiva, la población quedó establecida en 355 empresas.

Por otro lado, los datos necesarios para llevar a cabo esta investigación se han obtenido fundamentalmente a través de fuentes de información "primarias", es decir, a través de un cuestionario que fue remitido por correo postal y/o on-line a la totalidad de las 355 empresas seleccionadas. Para la elaboración del cuestionario, se realizó una primera versión analizando investigaciones previas relativas a la Norma ISO 9000 (Buttle, 1997; Casadesús et al., 2000; Escanciano et al., 2001; Casadesús et al., 2004; Mercado et al., 2005). Con posterioridad fue objeto de un "pretest" con expertos en esta materia de dos de las empresas participantes (Airbus España e lberia LAE Mantenimiento), con las que se mantuvo una entrevista en profundidad, redactándose a continuación el cuestionario definitivo para su envío a las empresas participantes. Una vez finalizado el proceso de recepción de cuestionarios cumplimentados, se concluye que el número de cuestionarios válidos es de 115 , lo que representa un $32,4 \%$ de la población.

En cuanto a la metodología empleada para el tratamiento de los datos recibidos, al ser una población pequeña se dificulta la utilización de análisis paramétricos, por lo que para analizar las relaciones entre las variables y obtener conclusiones sobre los objetivos propuestos para esta investigación, se ha utilizado estadística descriptiva, y se ha procedido a comparar las desviaciones típicas a través de un análisis ANOVA, para confirmar si existen diferencias significativas entre los resultados obtenidos por la implantación de dichas Normativas ISO 9000 y EN 9100.

\section{Gestión de la calidad en el sector aeroespacial}

En la actualidad, los altos niveles de competitividad y de globalización aplicables a casi todos los sectores, han provocado un amplio desarrollo del concepto de gestión de la calidad, como un enfoque estratégico para el tratamiento de la calidad en la empresa, que se sustenta en los principios de orientación al cliente, mejora continua, enfoque en las personas y visión global de la organización (Camisón et al., 2009). En este entorno, aparecen los Sistemas de Gestión de la Calidad (SGC), que permiten a las empresas dotarse de sistemas de gestión para establecer políticas y responsabilidades, asignar recursos e identificar actividades clave (Criado y Calvo, 2009). La implantación de un SGC se ve impulsada por la expectativa de beneficios que se derivan del mismo, ya que la mejora continua de la calidad de los productos ofertados por una empresa, debería aportar resultados positivos y medibles (Alvarez et al., 2013).

La industria aeroespacial siempre ha estado a la vanguardia del desarrollo 
de los SGC, siendo sus estándares muy superiores a los que se pueden encontrar en otros sectores industriales, salvo en el sector de la automoción (Gutiérrez, 2008), debido a la consideración de la seguridad como principal atributo que ha de poseer cualquier producto en esta industria. Por otro lado, algunas de las más recientes características de este sector, como la elevada subcontratación y dependencia de tecnología usada en otros sectores, así como la internacionalización, llevan a añadir aún más riesgo y complejidad a la gestión de la seguridad de los productos finales, por lo que se ha hecho fundamental el desarrollo de estándares comunes a todos los intervinientes, que serán descritos en la siguiente subsección.

\subsection{Evolución de la Gestión de la Calidad en el Sector}

El "esquema de autoevaluación" constituyó el primer paso en la evolución de los sistemas de calidad en la industria. Posteriormente, y aún hasta nuestros días, las empresas del sector han empleado los "esquemas de segunda parte", es decir, auditorias de los sistemas de calidad de los proveedores, en base a criterios y métodos propios de cada cliente, para analizar si un suministrador tiene o no capacidad para proveer un bien de manera constante y fiable de acuerdo a los requisitos que se establezcan en el contrato.

Buscando una mayor eficiencia, ya que con el esquema de segunda parte se podría incluso entorpecer el proceso productivo, debido al cumplimiento simultáneo de diferentes criterios, según cada una de las auditoras, en los años noventa se produce una tendencia hacia el desarrollo y adopción de sistemas basados en "esquemas de tercera parte". Dichos esquemas suponen la edición de unas Normas que contienen especificaciones técnicas basadas en los resultados de la experiencia y del desarrollo tecnológico fruto del consenso de todas las partes interesadas en la actividad, su cumplimiento debe ser certificado por una tercera entidad (Organismos de Certificación).

La autoridad le ha sido otorgada por las Entidades de Acreditación. Las Normas ISO 9000, se fueron expandiendo dentro del sector como el único SGC que debía ser considerado, y se constituyen en el ejemplo más extendido de estos esquemas de tercera parte. No obstante, esta Normativa no se ajustaba en su totalidad a las especificidades de la industria aeroespacial, por lo que la mayoría de las compañías más importantes del sector, continuaron produciendo sus propios suplementos a la Norma, con la consiguiente multiplicación de los requisitos para los suministradores, lo que provocaba de nuevo los problemas de los esquemas de segunda parte, citados en el párrafo anterior.

\subsection{La Normativa EN 9100}

En diciembre de 1998 los mayores suministradores, fabricantes y asociaciones comerciales de Europa, Asia y América se unieron formando el Internacional Aeroespacial Quality Group (IAQG por sus siglas en inglés). La creación del IAQG supuso un gran avance para la armonización, normalización y globalización de este sector, resolviendo de esta forma los problemas de multiplicidad de 
requisitos a cumplir por parte de los proveedores que intervienen en el mismo (Grijalbo y Prida, 2005a).

En el año 1999, el IAQG promovió la familia de Normas EN 9100 para el sector (AS 9100 en América y SIAC 9100 en Asia), con el objetivo de estandarizar los requisitos adicionales del sector a un nivel más concreto que ISO 9000 , buscando una reducción de la discrecionalidad, la mejora de la calidad y la reducción de los costos de la cadena de valor de la industria aeroespacial. En el año 2005, más de 160 empresas del sector a nivel mundial tenían la certificación EN 9100 (ATECMA, 2008). En diciembre del año 2012 la base de datos OASIS del IAQG, donde se registran las empresas que han implantado esta Normativa, contaba con 14.300 sedes de empresas certificadas a nivel mundial, de las cuales 448 correspondían a España ${ }^{3}$.

Las Normas EN 9100, en su versión original de 1999, incluían tanto los requisitos derivados de ISO 9000 (versión 1994), como 83 requisitos adicionales específicos de la industria aeroespacial, dando especial importancia a las áreas consideradas que podrían tener un mayor impacto en la seguridad y fiabilidad de los productos aeroespaciales, como son: diseño, compras, control de procesos, inspección y ensayo, y control de no-conformidades. El grupo de Normas EN 9100 de contenido genérico, emitidas por el IAQG, está constituido por las siguientes (IAQG, 2009):

- EN 9100 -Modelo para el Aseguramiento de Calidad en el Diseño, Desarrollo, -Producción, Instalación y Servicio Posventa.

- EN 9110 -Series Aeroespaciales, Modelo para el Aseguramiento de la Calidad aplicable a Organizaciones de Mantenimiento.

- EN 9120 -Series Aeroespaciales, Modelo para el Aseguramiento de la calidad en el almacenamiento.

En el Cuadro 1 se recogen las principales novedades de este grupo de Normas EN 9100 respecto a la Normativa ISO 9000.

\section{Las Normas de Aseguramiento de la Calidad: estudios previos sobre los resultados de su implantación}

Los trabajos existentes en materia de la Normativa EN 9100 no son muy numerosos. Algunos se centran en una descripción de las características generales de esta Normativa, así como en sus principales diferencias respecto a la familia de Normas ISO 9001 (Beltrán, 2002; Juny, 2005; Grijalbo y Prida, 2005b; Gutiérrez, 2008); otros detallan el nivel de implantación de las Normas EN 9100, que si bien es general en los grandes fa-

3 Hay que destacar que en la Base de Datos OASIS figuran sedes certificadas, por lo que una misma empresa con varias sedes figurará en más de una ocasión (lo que suele ser habitual en el caso de las empresas grandes). Por este motivo el número de certificaciones de dicha base supera el número total de empresas de este sector tanto a nivel mundial como español. 


\title{
Cuadro 1 \\ Principales novedades Normas EN 9100 frente a ISO 9000
}

\author{
CAPITULO 4. SISTEMA DE GESTIÓN DE LA CALIDAD
}

Apartado 4.2 Requisitos de la documentación

4.2.1. La organización asegurará que el personal tenga acceso a la documentación del SGC y conozca los procedimientos relevantes, así como que los clientes y/o las autoridades reguladoras tengan acceso a la documentación del SGC.

4.2.2. Clara relación entre los requisitos de la Norma y los procedimientos documentados por la empresa.

4.2.3. La organización coordinará los cambios en los documentos con los clientes y/o autoridades reguladoras de acuerdo a requisitos del contrato o reglamentarios.

4.2.4. Los métodos de control de registros deben estar definidos, así como que todos los registros deben estar disponibles para su revisión de acuerdo con el contrato o requisitos reglamentarios.

\section{CAPITULO 5. RESPONSABILIDAD DE LA DIRECCIÓN}

Apartado 5.5. Responsabilidad, autoridad y comunicación

5.5.2. El representante de la dirección tendrá libertad organizativa para resolver asuntos relativos a la calidad.

\section{CAPITULO 6. GESTIÓN DE LOS RECURSOS}

Apartado 6.4. Ambiente de trabajo

Se describen los factores que pueden afectar a la conformidad del producto, como temperatura, humedad, entre otros.

CAPITULO 7. REALIZACIÓN DEL PRODUCTO

Apartado 7.1. Planificación de la realización del producto

Se requiere la identificación de los recursos para soportar la operación y mantenimiento del producto.

Apartado 7.2. Procesos relacionados con el cliente

7.2.2. Se requiere la evaluación de riesgos (nueva tecnología y/o entrega en tiempos cortos).

Apartado 7.3. Diseño y desarrollo

7.3.1. Se aclaran los requisitos para la planificación y la designación de responsabilidades en las diferentes etapas del diseño.

7.3.3. Las características clave deben estar identificadas. Así mismo, se requiere que todos los datos para permitir que el producto sea identificado, fabricado, inspeccionado, utilizado y mantenido, sean definidos por la organización.

7.3.4. Se requiere autorización formal para el progreso hacia las siguientes etapas del diseño.

7.3.5. Se identifican algunos ejemplos de actividades de verificación del "Diseño y desarrollo"

7.3.6. Se aclaran aspectos de la validación del "Diseño y desarrollo". Las evidencias documentales deben demostrar que la definición del producto se ajusta a los requerimientos especificados. Así mismo se indica que donde las pruebas para la verificación y validación son necesarias, éstas se planificarán, controlarán, revisarán y documentarán, con el fin de asegurar y demostrar una serie de requerimientos.

7.3.7. Se requiere la aprobación por el cliente y/o autoridad, cuando exista un requisito contractual o reglamentario. 


\section{Cuadro 1 (Continuación)}

\section{CAPITULO 7. REALIZACIÓN DEL PRODUCTO}

Apartado 7.4. Compras

7.4.1. La organización deberá ser responsable de la calidad de todos los productos comprados, incluidas las fuentes designadas por el cliente. Se añaden nuevos requerimientos: mantenimiento del registro de proveedores aprobados, revisión periódica del rendimiento del proveedor, mecanismos a desarrollar con aquellos que tengan pobres rendimientos, así como con fuentes de proceso especiales aprobadas por el cliente, y determinación expresa de la autoridad de la empresa para desaprobar el uso de las fuentes.

7.4.2. Se detalla información adicional a incluir como información de compra.

7.4.3. Se suministran ejemplos de actividades de verificación de productos comprados y algunos requerimientos adicionales específicos para asegurar que sólo el material conforme está correcto para su uso. Se exige la validación periódica de los informes de las pruebas para materias primas, cuando las actividades de verificación estén delegadas en el proveedor. Éstas actividades deberán permitir al cliente verificar el producto en las instalaciones del proveedor cuando se especifique en el contrato.

\section{Apartado 7.5. Producción y prestación de servicio}

7.5.1. Se detallan algunas consideraciones de la planificación de la producción/provisión de servicio, como características clave y procesos especiales. Se especifican algunas de las condiciones controladas para llevar a cabo la producción, las cuales deberán ser incluidas en la planificación, así como la evidencia de responsabilidades, de inspección y realización de todas las operaciones, de prevención de objetos extraños y de control de las utilidades, así como criterios de la calidad del trabajo, de tal forma que se basen en normas escritas y muestras representativas.

Se requiere que las operaciones de producción se lleven a cabo de acuerdo con los datos aprobados. Así mismo se exigen controles apropiados para los cambios de procesos, como la identificación de personal autorizado, la aprobación del cliente y/o autoridad, documentar los cambios y su evaluación para confirmar la consecución de los efectos deseados.

Se especifican los requerimientos para el control y validación de los equipos de producción, herramientas y utillajes, entre otros. Así mismo se detallan requerimientos para el control del trabajo transferido provisionalmente fuera de las instalaciones de la organización.

Se especifican los requerimientos para el control de las actividades de servicio.

7.5.2. Se clarifica la forma de implementar los requerimientos existentes para la validación de los procesos.

7.5.3. Se detalla la identificación de la configuración del producto, con el fin de identificar cualquier diferencia entre la configuración real y la acordada. Además cuando se utilicen medios de reconocimiento de la aceptación (sellos), la organización deberá establecer y documentar controles sobre dichos medios.

7.5.5. Se detallan los requerimientos de preservación del producto incluyendo limpieza, manipulación, control de vida de la estantería y rotación del stock. 


\section{Cuadro 1 (Continuación)}

\section{CAPÍTULO 8. MEDICIÓN, ANÁLISIS Y MEJORA}

\section{Apartado 8.1. Generalidades}

Se clarifica como pueden ser usadas las técnicas estadísticas y para que propósito.

\section{Apartado 8.2. Seguimiento y medición}

8.2.2 Se especifican las herramientas y técnicas (ejemplo, check-list), que deben ser desarrollados para apoyar el proceso de auditoría, que deberán ser revisadas periódicamente para analizar su efectividad. Se exige que las auditorias deben también satisfacer los requisitos contractuales y/o /reglamentarios.

8.2.3. Se detallan requerimientos de aplicación en el caso de procesos de no conformidad.

8.2.4. Se incluyen indicaciones para el seguimiento y medición del producto, como el control y seguimiento de las características claves, usando inspección por muestreo validado estadísticamente. Se especifica que no se utilizará ningún producto sin inspección o bajo procedimientos de recuperación positiva.

Se clarifican los requerimientos de la medición, incluyendo criterios de aceptación, secuencia de las operaciones, registros a ser creados e instrumentos a utilizar.

\section{Apartado 8.3. Control del producto no conforme}

Se especifica que las responsabilidades para la revisión y disposición de un producto no conforme deben de estar definidas y documentadas, de tal forma que una pieza recuperada para el uso después de estar inutilizada, no pueda ser utilizada sin la autorización del cliente. Se indica que la chatarra debe de ser marcada de forma clara y permanente o estar físicamente inutilizable.

\section{Apartado 8.5. Mejora}

8.5.2. Se fijan requisitos para las acciones correctoras de los suministradores, cuando se determine que son responsables de las deficiencias detectadas.

Fuente: Elaboración propia.

bricantes y suministradores del sector, es más desigual en proveedores de menor tamaño (Grijalbo y Prida, 2005a y 2005c); otros estudios recogen modelos y pautas para realizar la implantación de esta Normativa (Taddei, 2007; Hopsort y Frances, 2011); finalmente en otros trabajos se encuentran descripciones de la forma en que se ha llevado a cabo la implantación en ciertas empresas aeronáuticas (Murga, 2002; Vilar, 2003; Mugarra, 2003).

Dado que ninguno de estos estudios previos sobre las Normas EN 9100 se centra en los aspectos que se pretenden en los objetivos de esta investiga- ción, se ha procedido a revisar las principales investigaciones existentes sobre las Normas ISO 9000. Estas han sido objeto de múltiples estudios, si bien ninguno de ellos ha hecho referencia al sector aeroespacial de forma específica es la predecesora de EN 9100 en el sector aeroespacial como norma de aseguramiento de la calidad, y teniendo en cuenta el gran nivel de implantación de ISO 9000 en dicho sector, se ha considerado que las conclusiones de estos estudios sobre los beneficios de la implantación de ISO 9000 constituían un adecuado punto de partida para la presente investigación. 
En lo referente a las principales investigaciones que de forma específica 0 entre otros temas tratan sobre los resultados derivados de la implantación de ISO 9000, Mann y Kehoe (1994) señalan que valorar los efectos de las iniciativas de calidad es muy difícil debido a que muy pocas empresas son capaces de aislar dichos efectos, o bien de medirlos cuantitativamente. No obstante lo anterior, es numeroso el grupo de estudios que han analizado, no sin dificultad y con heterogeneidad de resultados, las consecuencias de la implantación de la Normas ISO 9000.

En general se aprecia que una mayoría de los estudios concluye que la Normativa ISO 9000 supone efectos beneficiosos para las empresas; no obstante lo anterior, y aunque sean minoría, existen otros estudios que no ponen de manifiesto dichos efectos positivos, o al menos no en todos los casos o circunstancias, tal y como indican Terziosvski et al., (1997), Gavin (2000), Singles et al., (2001), Wayhan et. al., (2002); Martínez-Costa y Martínez-Lorente (2007), Martínez-Costa et al., (2009) y Boiral y Amara (2009). Todo esto parece indicar que no basta con obtener la certificación asociada con estas Normas para lograr sus beneficios potenciales, sino que existen determinados factores que pueden condicionar dichos resultados positivos (Lee et al., 2009; Psomas et al., 2010; Boiral, 2011)

En cuanto a los resultados positivos generados por la adhesión a la Norma, se debe indicar que diversos trabajos (Tsiotras y Gotzamani, 1996; Vloeberghs y Bellens, 1996; Escanciano et al., 2001; Douglas et al., 2003; Casadesus et al., 2004; Sampaio et al., 2009; Tari et al., 2012), los clasifican en beneficios relativos a: "aspectos internos" $y$ "aspectos externos", En el Cuadro 2, se detallan los aspectos más resaltantes:

\section{Cuadro 2 \\ Desglose de los resultados positivos derivados de la adhesión a ISO 9000}

\section{ASPECTOS INTERNOS}

\section{Relativos a procesos organizativos}

-Control sobre la gestión de la producción, establecimiento de responsabilidades y regla, documentación de los procesos, entre otros.

\section{Relativos a la ejecución de operaciones}

-Mejor utilización de recursos, reducción costos de inspección, disminución de costos logísticos, y disminución de no conformidades.

\section{Relativos a la gestión de RRHH}

-Satisfacción en el trabajo, funcionamiento de los equipos de trabajo y sistemas de sugerencias de empleados.

\section{ASPECTOS EXTERNOS}

Relativos a aspectos financieros y comerciales

-Volumen de ventas, cuota de mercado, y ratio ventas por empleado.

Relativos a la relación con los clientes

-Retención de clientes, quejas recibidas e imagen en el mercado.

Fuente: Elaboración propia. 
En este sentido, existen investigaciones que ponen de manifiesto los beneficios que aporta la implantación de la Norma ISO 9000 se refieren fundamentalmente a aspectos internos (Brown et al., 1998; Climent, 2005; Bhuyam y Alam, 2005; Bayati and Taghavi, 2007; Terziovsky y Power, 2007; Martinez-Costa et al., 2008; Lo et al., 2009; Wahid y Corner, 2009); sin embargo, otros estudios ponen el énfasis en los beneficios relativos a aspectos externos (Hendricks y Singhal, 1996; Wayhan et al., 2002; Nicolau y Sellers, 2003; Martínez-Costa y Martínez-Lorente, 2007; Corbett et al., 2005; Sharma, 2005; Terlaak y King, 2006; Vinuesa, 2007; Benner y Veloso, 2008; Dick et al., 2008); aunque la mayoría pone de manifiesto resultados positivos tanto de índole interna (relativos a los procesos organizativos, ejecución de operaciones y gestión de recursos humanos), así como externa, es decir, relativos a aspectos financiero-comerciales y de gestión de la relación con los clientes (Tsiotras y Gotzamani, 1996; Buttle, 1997; Hareton et al., 1999; Casadesús y Giménez, 2000; Romano, 2000; Casadesús y Heras, 2001; Escanciano et al., 2001; Santos y Escanciano, 2002; Casadesús et al., 2004; Mercado et al., 2005; Rodriguez et al, 2006; Calisir, 2007; Lo y Chang, 2007; Zaramdini, 2007; Feng et al., 2008; Jang y Lin, 2008).

Por otro lado, Tari et al., (2012) llevan a cabo una revisión de 82 investigaciones previas en relación con los beneficios generados por la adhesión a ISO 9000, llegando a la conclusión que los efectos positivos más citados en dichos estudios, son los aspectos internos relativos a la ejecución de operaciones y a los recursos humanos y entre los aspectos externos, los relacionados con los clientes.

\section{Normas de Aseguramiento de la Calidad en el sector aeroespacial español: resultados de su implantación}

En base a los resultados obtenidos en esta investigación, se debe indicar que ambas Normativas ISO 9000 y EN 9100 están ampliamente implantadas en las entidades que han cumplimentado el cuestionario (111 y 80 de las empresas participantes respectivamente). Con una antigüedad desde la adhesión superior a los tres años del $96 \%$ de las empresas para ISO 9000, y del $86 \%$ para EN 9100. El nivel de intensidad en la implantación también es elevado, ya que en el caso de ISO 9000 el $78 \%$ de las empresas ha adherido todos sus procesos a dicha Normativa, siendo el porcentaje similar para EN 9100 (77\%). Ambos aspectos, refuerzan la validez de las respuestas obtenidas a través de los cuestionarios recibidos que indican aspectos técnicos del trabajo realizado.

La población estuvo conformada por 335 empresas del sector aeroespacial español. Se empleó el muestreo aleatorio como técnica, obteniendo una muestra de 115 cuestionarios recibidos, los cuales fueron enviados mediante encuesta postal y on line a los directores o responsables de calidad de las entidades estudiadas. Se asume un nivel de confianza del $95 \%(Z=1,96 ; p=q=0,5)$ y un error muestral de 7,5\%. Los datos se recopilaran desde el año 2009 - 2010.

A partir de las respuestas de las 111 empresas del sector que han implantado ISO 9000, se ha elaborado el siguiente gráfico que recoge el porcentaje de las mismas. La escala empleada consta de cuatro 
opciones progresivas de contestación: 1) nada relevante; 2 ) poco relevante; 3 ) bastante relevante y 4) muy relevante.

Al indagar sobre la valoración de la influencia de la implantación de la normativa ISO 9000 considerando los beneficios antes identificados (cuadro 2), los resultados se orientan a las alternativas "bastante relevante" o "muy relevante" cada uno de los efectos percibidos (tanto internos como externos) fruto de la implantación de ISO 9000 (Gráfico 1).

Se pone de manifiesto que en cuanto a los beneficios de tipo "interno", las empresas participantes manifiestan una influencia positiva de la adhesión a ISO 9000, aunque no de forma clara en todas sus modalidades. Se puede destacar las mejoras de tipo "organizativo" con un $67 \%$ de las entidades participantes que las señalan, seguidas de las relacionadas con la "ejecución de las operaciones" (65\% de entidades), sin embargo en lo referente a efectos positivos sobre la gestión de los "recursos humanos", sólo un $43 \%$ de las entidades participantes así lo han señalado. Con respecto a los beneficios de tipo "externo", las empresas participantes ponen de manifiesto que implantar ISO 9000 tiene una mayor influencia positiva para su obtención; en este caso se obtienen porcentajes elevados de empresas que así lo señalan, tanto en las mejoras "financiero-comerciales" $(78 \%)$ como en lo relativo a la "relación con clientes" (77\%).

Según estos datos las empresas participantes consideran que implantar la Normativa ISO 9000 tiene una mayor influencia para la obtención de beneficios sobre aspectos "externos" (financieroscomerciales y de gestión de la relación con los clientes), que sobre aspectos "internos" (procesos organizativos, ejecución de operaciones y gestión de recursos humanos). Cualquiera de los benefi-

\section{Gráfico 1 \\ Efectos positivos "internos" y "externos" derivados de la adhesión a ISO 9000}

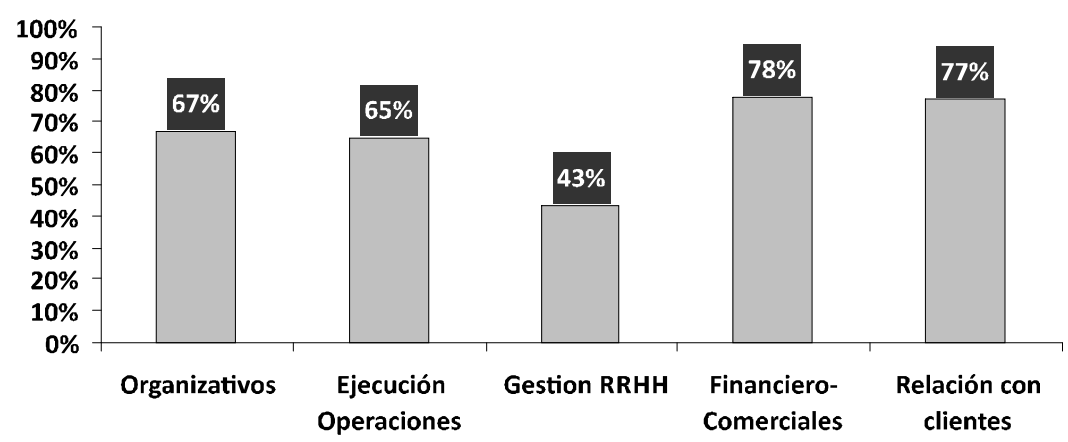

TOTAL ISO 9000

Fuente: Elaboración propia. 
cios de tipo "externo" (presentan un mínimo del $77 \%$ de las entidades que los señalan), supera a cualquier beneficio de tipo "interno" (presentan un máximo del $67 \%$ de ellas).

En definitiva, los resultados obtenidos sobre la implantación de ISO 9000 en el sector aeroespacial español, están en la línea de lo establecido por Tsiotras y Gotzamani (1996), Buttle (1997), Hareton et al., (1999), Casadesús y Giménez (2000), Romano (2000), Casadesús y Heras (2001), Escanciano et al., (2001), Santos y Escanciano, 2002; Casadesús et al.,, 2004; Mercado et al., 2005; Rodriguez et al, 2006; Calisir, 2007; Lo y Chang, 2007; Zaramdini, 2007; Feng et al., 2008; Jang y Lin, 2008), en el sentido de que la implantación de dicha Normativa provoca efectos positivos tanto en lo que respecta a "aspectos internos" como "externos".

Por otra parte se planteó indagar sobre la valoración EN 9100, para la apa- rición de beneficios sobre los distintos aspectos identificados en el Cuadro 2.

Las respuestas aportadas por las 80 empresas del sector que han implantado la Normativa EN 9100, permitió elaborar el Gráfico 2, que recoge los porcentajes de empresas que han reconocido como "bastante relevante" o "muy relevante" cada uno de los efectos percibidos (tanto internos como externos), como consecuencia de la implantación de EN 9100.

El Gráfico 2 muestra que según las respuestas de las empresas participantes, la adhesión a EN 9100 puede favorecer la aparición de beneficios de tipo "interno". Dentro de estos beneficios destacan las mejoras "organizativas" $(78 \%$ de las entidades), seguidas de las relacionadas con la "ejecución de las operaciones" (68\% de entidades), y no tanto los efectos sobre los "recursos humanos", ya que sólo un $51 \%$ de ellas lo han seleccionado. Por otro lado, según los cuestionarios re-

\section{Gráfico 2 \\ Efectos positivos "internos" y "externos" derivados de la adhesión a EN 9100}

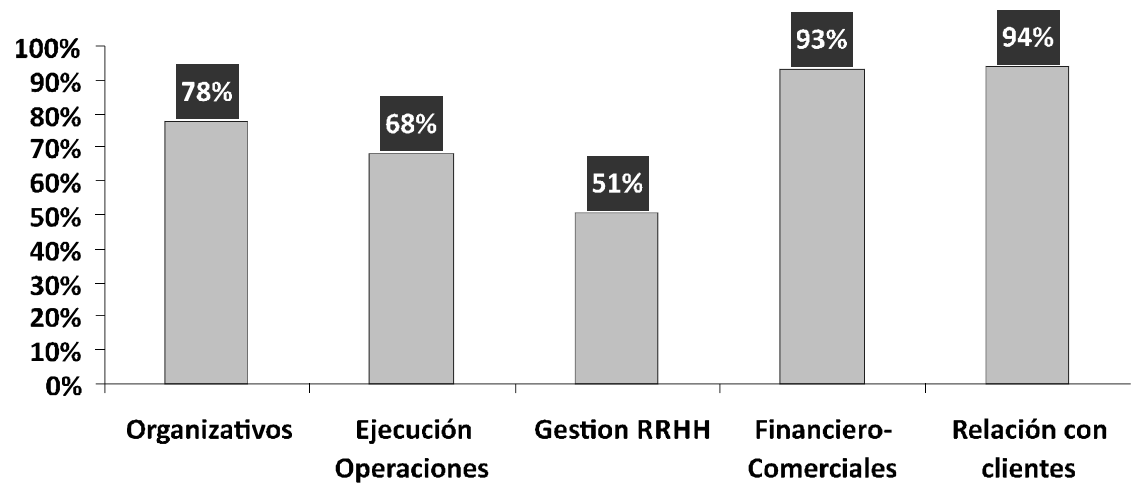

TOTAL EN 9100

Fuente: Elaboración propia. 
cibidos, la implantación de EN 9100 puede tener una mayor influencia para la aparición de beneficios sobre aspectos "externos". En este caso se obtienen porcentajes elevados tanto en las mejoras "financiero-comerciales" (93\%) como en lo relativo a la "relación con clientes" (94\%). Así mismo, los beneficios sobre aspectos "externos" que pueden aparecer fruto de la Normativa EN 9100 superan a los de tipo "interno", ya que cualquiera de los beneficios "externos", es señalado por un porcentaje de entidades superior al de cualquier beneficio de tipo "interno" (93\% es el porcentaje mínimo de entidades que presentan los aspectos "externos", mientras que el $78 \%$ es el máximo de los aspectos "internos").

Estos resultados ponen de manifiesto que la adhesión a EN 9100 en el sector aeroespacial español, provoca resultados positivos en relación tanto con "aspectos internos" como "externos", en la misma línea de lo que indican Tsiotras y Gotzamani (1996), Buttle (1997), Hareton et al., (1999), Casadesús y Giménez (2000), Romano (2000), Casadesús y Heras (2001), Escanciano et al., (2001), Santos y Escanciano, 2002; Casadesús et al., 2004; Mercado et al., 2005; Rodriguez et al, 2006; Calisir, 2007; Lo y Chang, 2007; Zaramdini, 2007; Feng et al., 2008; Jang y Lin, 2008) para la Normativa ISO 9000 , referencia que se ha tenido que utilizar, al no existir estudios previos sobre los resultados de EN 9100 con los que poder realizar una comparación.

Al comparar los resultados derivados de la implantación de las normativas EN 9100 e ISO 9000, como objetivos básicos de la investigación, se procedió a considerar dos grupos de empresas: por un lado aquellas del sector que han implantado EN 9100 (80 empresas), y por otro aquellas otras que solamente han implantado ISO 9000 (31 empresas).

En el Gráfico 3 se recoge el análisis comparativo de los porcentajes de empresas de cada grupo considerado, que han reconocido como "bastante relevante" o "muy relevante" cada uno de los efectos positivos percibidos (tanto internos como externos), como consecuencia de la implantación de la Normativa EN 9100 o ISO 9000 según el grupo de empresas de que se trate en cada caso.

Las empresas participantes en esta investigación ponen de manifiesto que la implantación de la Normativa EN 9100 provoca unos beneficios superiores a los derivados de ISO 9000 (en todos los aspectos analizados la Normativa EN 9100 genera un mayor impacto positivo que ISO 9000), destacando las diferencias en lo relativo a los efectos positivos "internos" relacionados con los procesos organizativos (33 puntos porcentuales de diferencia), y a los efectos positivos "externos" relativos a la gestión de la "relación con la clientela" (42 puntos porcentuales de diferencia).

Para confirmar estas diferencias de efectos positivos tanto "internos" como "externos" se ha realizado un análisis de la varianza ANOVA. Mediante este análisis se comparará si los efectos positivos que aparecen en el primer grupo de empresas fruto de su adhesión a la Normativa EN 9100, son significativamente distintos de los que se generan en el segundo grupo de empresas como consecuencia de la implantación de ISO 9000. Los resultados se muestran en la Tabla 1. 


\section{Gráfico 3}

Análisis comparativo de los efectos positivos "internos" y "externos" derivados de la adhesión a EN 9100 versus ISO 9000

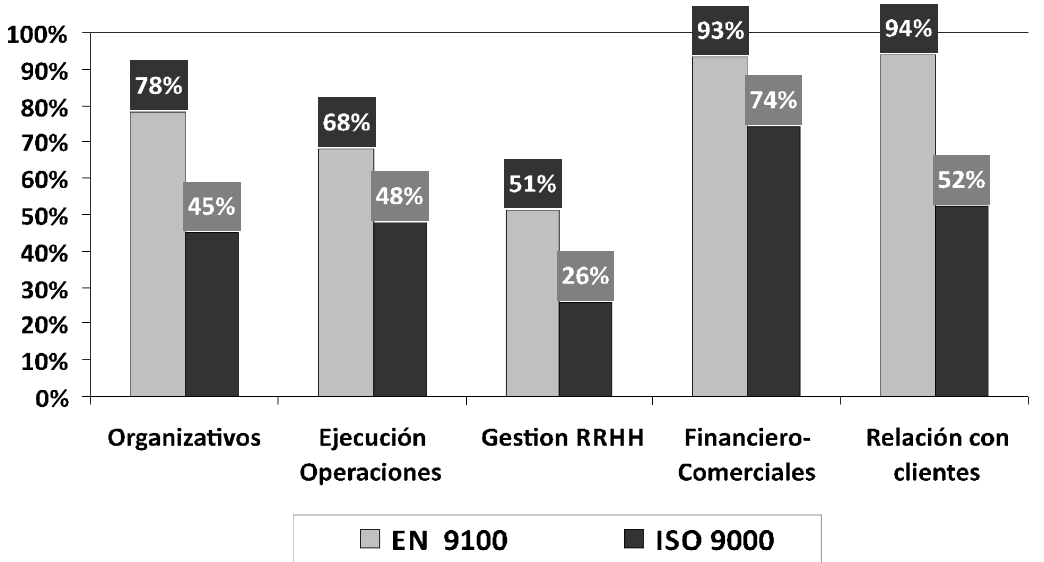

Fuente: Elaboración propia.

Tabla 1

ANOVA de los efectos positivos "internos" y "externos" derivados de la adhesión a la Normativa EN 9100 versus ISO 9000

\begin{tabular}{lc}
\hline \multicolumn{1}{c}{ Variables } & Sig. \\
\hline Efectos internos organizativos & 0,017 \\
Efectos internos de ejecución de operaciones & 0,038 \\
Efectos internos relativos a RRHH & 0,000 \\
Efectos externos financieros y comerciales & 0,005 \\
Efectos externos relacionados con clientes & 0,000 \\
p<0,05 es significativo al 95\% & \\
Factor: implantación normativa EN 9100 O ISO 9000
\end{tabular}

Fuente: Elaboración propia.

El análisis de la varianza respecto a los distintos tipos de efectos positivos "internos" y "externos" considerados, confirma con niveles de significación menores a 0,05, la existencia de distintos patrones de comportamiento entre las empresas del sector aeroespacial español que han implantado la Normativa EN 9100, y las que solamente se han adherido a ISO 9000.

Por otro lado, para completar este análisis comparativo, se ha planteado indagar sobre la evolución de sus indicadores de calidad en los últimos 5 años. Las entidades participantes disponían de las siguientes opciones progresivas de con- 
testación): 1) ha empeorado sensiblemente; 2) ha empeorado levemente; 3) se mantuvo similar; 4) ha mejorado levemente; 5) ha mejorado sensiblemente.

Con el fin poder realizar el análisis comparativo entre los resultados obtenidos por las empresas del sector aeroespacial español, al implantar las normas de aseguramiento de la calidad citadas (ISO 9000 y EN 9100), en este caso mediante el estudio de la evolución de sus indicadores de calidad en los últimos cinco años, se vuelven a considerar los dos grupos de empresas anteriores: por un lado aquellas empresas del sector que han implantado EN 9100 (80 empresas), y por otro aquellas otras que solamente han implantado ISO 9000 (31 empresas).

En el Gráfico 4, se ponen de manifiesto los resultados obtenidos a partir de las contestaciones de las empresas participantes, en cuanto a la evolución de los indicadores de calidad relacionados tanto con mejoras "internas" como "externas", reflejando los porcentajes de empresas de cada uno de los dos grupos considerados (adheridos a la Normativa EN 9100 o ISO 9000 respectivamente), que han reconocido que dichos indicadores han mejorado "levemente" o "sensiblemente".

Se evidencia (Gráfico 4) que todos los indicadores de calidad analizados, tanto los relativos a aspectos "internos" como "externos", han evolucionado más positivamente en el grupo de empresas que se han adherido a la familia de Normas EN 9100, en comparación con las que solamente se han adherido a la Normativa ISO 9000. Aunque en todos los casos se observan diferencias significativas, son especialmente destacables las relativas a los indicadores "internos" relativos a los procesos organizativos, así como a los "externos" relacionados con aspectos "financieros y comerciales.

\section{Gráfico 4}

Indicadores de calidad sobre aspectos "internos" y "externos" en los grupos de empresas que han implantado la Normativa EN 9100 o ISO

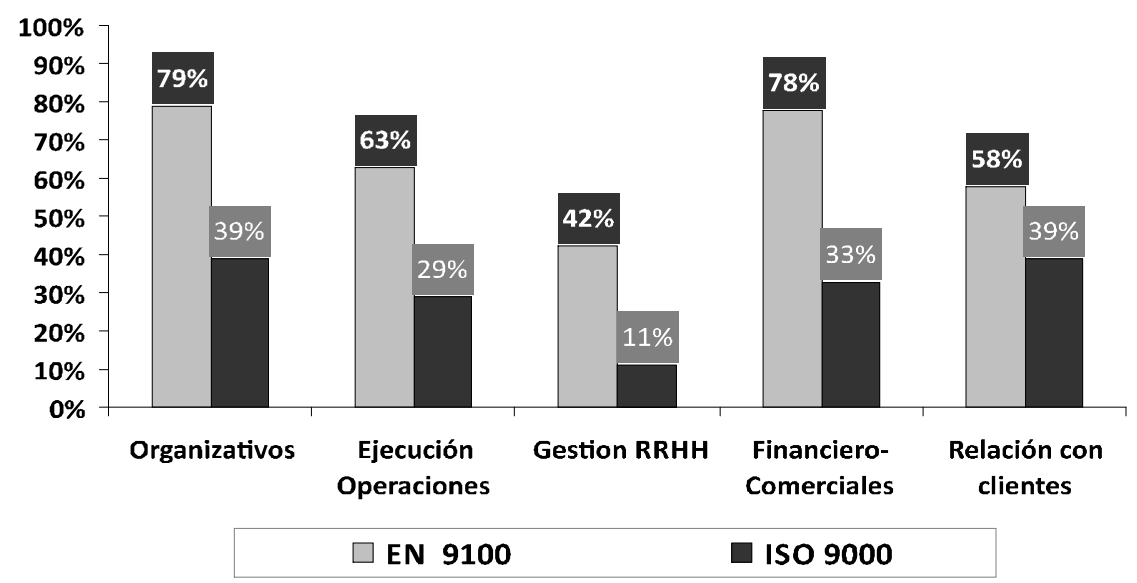

Fuente: Elaboración propia. 
Para confirmar estas diferencias en cuanto a la evolución de los indicadores de calidad tanto "internos" como "externos" entre el grupo de empresas del sector que han implantado EN 9100 y el grupo de las que solamente se han adherido a la Normativa ISO 9000, se ha realizado un análisis de la varianza ANOVA. Mediante este análisis se ha comparado si la evolución de los indicadores de calidad del grupo de empresas que han implantado la Normativa EN 9100, es significativamente distinta de la evolución de dichos indicadores para el grupo de las empresas del sector que solamente se han adherido a ISO 9000 . Los resultados se muestran en la Tabla 2.
EN 9100, y el grupo de las que solamente se han adherido a ISO 9000.

En resumen, los Gráficos 3 y 4, así como las Tablas 3 y 4 , permiten dar respuesta al objetivo de esta investigación, al poner de manifiesto que la implantación de la Normativa EN 9100 en las empresas del sector aeroespacial español, provoca unos beneficios superiores a los derivados de la adhesión a las Normas ISO 9000, tanto en lo que se refiere a aspectos de tipo "interno" como "externo", compensando de esta manera los mayores costos de implantación y mantenimiento de dicha Normativa.

\section{Tabla 2}

ANOVA de la evolución de los indicadores de calidad sobre aspectos "internos" y "externos"

\begin{tabular}{lc}
\hline \multicolumn{1}{c}{ Variables } & Sig. \\
\hline Indicadores de calidad internos organizativos & 0,000 \\
Indicadores de calidad internos de ejecución de operaciones & 0,005 \\
Indicadores de calidad internos relativos a RRHH & 0,000 \\
Indicadores de calidad externos financieros y comerciales & 0,000 \\
Indicadores de calidad externos relacionados con clientes & 0,004 \\
p<0,05 es significativo al 95\% & \\
Factor: implantación normativa EN 9100 O ISO 9000 & \\
\hline
\end{tabular}

Fuente: Elaboración propia.

El análisis de la varianza respecto a los indicadores de calidad relativos a todos los aspectos "internos" y "externos", muestra con niveles de significación menores a 0,05 , la existencia de distintos patrones de comportamiento entre el grupo de empresas del sector aeroespacial español que han implantado la Normativa

\section{Conclusiones}

La aportación de esta investigación se concreta en dos puntos: a) en primer lugar, se analizan los resultados derivados de la implantación de las Normas de Aseguramiento de la Calidad (ISO 9000 y EN 9100) en el sector aeroespacial, que hasta la fecha 
no había sido objeto de análisis en investigaciones precedentes, más allá de ciertos estudios genéricos y sobre procesos formales de implantación relativos a las Normas EN 9100; $y$ b) en segundo lugar, y dado que en este sector existe una Normativa específica de aseguramiento de la calidad (EN 9100), que surge como desarrollo de las Normas genéricas ISO 9000, se ha llevado a cabo un análisis comparativo de los resultados obtenidos a partir de la adhesión a dichas Normas (ISO 9000 y EN 9100), por parte de las empresas del sector aeroespacial español.

En cuanto al análisis individual de los beneficios derivados de la adhesión a estas Normativas en este sector, los resultados obtenidos en esta investigación para ambas Normativas, siguen la misma línea de estudios previos realizados sobre la Normativa ISO 9000, ya que las empresas participantes en este estudio, consideran mayoritariamente que la implantación tanto de EN 9100 como de ISO 9000 tiene una influencia positiva para la obtención de efectos positivos "internos" (relativos a la mejora de los procesos organizativos y de la ejecución de operaciones, así como de la gestión de los recursos humanos), y sobretodo "externos" (relativos a magnitudes financiero-comerciales, y de mejora de la gestión de la relación con los clientes). No obstante lo anterior, los efectos positivos "internos" relativos a la mejora de la "gestión de recursos humanos", presentan un porcentaje más reducido de empresas que identifica esta influencia positiva.

Estos primeros resultados de la investigación, donde a pesar de obtenerse beneficios internos y externos, predominan éstos últimos, pueden derivarse de las características intrínsecas del sector aeroespacial, donde predomina la internacionalización y la complejidad de los proyectos a desarrollar, de tal forma que lo habitual es que en la ejecución de un pedido estén involucradas empresas de distintos países y sectores, por lo que la adhesión a estas Normas de Aseguramiento de la Calidad es la única forma de garantizar frente al resto de los participantes del proyecto, así como frente a los clientes, la homogeneidad de los procesos y el logro individual de unos estándares de calidad, de tal forma que al ensamblar el producto final se logren los requerimientos de calidad y seguridad exigidos por el cliente. Por lo tanto, la implantación de estas Normas, además de proporcionar mejoras de la calidad interna de la empresa, se convierte en un factor crítico para poder participar en este mercado, lo que beneficia las relaciones con los clientes, así como en último término las magnitudes financiero-comerciales.

Por otro lado, la menor influencia positiva de la adhesión a estas Normas respecto a la "gestión de recursos humanos", puede derivarse de que la complejidad y la especialización de los productos de este sector, siempre ha exigido de todos los integrantes de sus empresas, un elevado compromiso con los requisitos de calidad y seguridad de sus productos, de tal forma que el margen de mejora a partir de la implantación de estas Normas es menos amplio.

En cuanto al análisis comparativo sobre los beneficios obtenidos en las empresas de este sector, según la Normativa de Aseguramiento de la Calidad implantada en cada caso, las respuestas de las empresas participantes en la investi- 
gación ponen de manifiesto que la implantación de la Normativa EN 9100 provoca unos efectos positivos sensiblemente superiores a los derivados de ISO 9000 (en todos los aspectos analizados la adhesión a EN 9100 genera un mayor impacto positivo que ISO 9000). Destacan especialmente las diferencias en lo relativo a los efectos positivos "internos" relacionados con la mejora de los procesos organizativos, y a los efectos positivos "externos" relativos a una mejor gestión de la "relación con la clientela".

Por otro lado, este análisis comparativo de los resultados positivos de ambas Normativas, se ha completado mediante el estudio de la evolución de los indicadores de calidad en los últimos cinco años, de los dos grupos de empresas considerados. El estudio realizado muestra que la evolución de todos los indicadores de calidad tanto "internos" como "externos", es sensiblemente mejor en el grupo de empresas que han implantado la Normativa EN 9100, en comparación con el grupo de empresas que solamente se han adherido a ISO 9000, destacando especialmente en este caso, la diferencia relativa a los aspectos internos de mejora de "procesos organizativos", y los externos relacionados con magnitudes "financiero-comerciales".

Todo lo anterior pone de manifiesto que, aunque ambas Normativas suponen efectos positivos para las empresas del sector aeroespacial español, la implantación de la Normativa específica EN 9100 provoca unos beneficios tanto de tipo interno como externo, superiores a las Normas ISO 9000. Estos resultados pueden derivarse del carácter más genérico de las Normas ISO 9000, acrecentado a partir de la versión ISO
9000:2000, en la que se introduce un mayor nivel de flexibilidad en su implantación, para poder extenderse con mayor facilidad al sector servicios y a las pequeñas y medianas empresas, que eran dos de las limitaciones de las versiones anteriores de 1987 y 1994 de estas Normas. Fruto de estos cambios y esta mayor flexibilidad en su aplicación, ciertos sectores industriales como el del automóvil (Norma ISO/TS 16949) o el aeroespacial (Norma EN 9100), se vieron obligados a elaborar una norma más adaptada a los requerimientos de los mismos, como forma de preservar los beneficios de los esquemas de evaluación de la calidad de "tercera parte" en las relaciones clienteproveedor, ya que para combatir el carácter general de las Normas ISO 9000, algunos clientes empezaban a solicitar individualmente el cumplimiento de requisitos adicionales de calidad, por encima de los establecidos en las Normas ISO 9000 , con el incremento de costes que esto suponía para los proveedores.

En definitiva, los requerimientos adicionales que contempla la serie de Normas EN 9100, especialmente adaptados a la problemática del sector aeroespacial, y que completan los genéricos de la Normativa ISO 9000, provocan un mayor nivel de efectos positivos en las empresas que implantan dichas Normas, sin que en este estudio se haya profundizado en cuales de dichos requerimientos adicionales tienen una mayor influencia para esta diferencia, lo que debería ser objeto de estudio en posteriores investigaciones de tipo cualitativo. Estos mayores beneficios permiten compensar el mayor coste inicial y de mantenimiento que EN 9100 supone para cumplir con dichos requisitos complemen- 
tarios, lo que para la gestión de estas empresas debería implicar una generalización de la implantación y la profundización en el desarrollo y asimilación interna de esta Normativa, buscando el logro de sus potenciales efectos positivos.

\section{Referencias Bibliográficas}

Alvarez, José; Fraiz, José Antonio y Del Río, María (2013). Implantación de un sistema de gestión de la calidad: beneficios percibidos. Revista Venezolana de Gerencia, Vol. 18, No. 63, pp. 379-407.

Anderson, Shannon; Daly, Daniel y Johnson, Marylin (1999). Why firms seek ISO 9000 certification: regulatory compliance or competitive advantage. Production and Operations Management, Vol. 8, No. 1, pp. 28-43.

Asociación Española de Constructores de Material Aeroespacial - ATECMA(2008). Memoria Anual.

Bayati, Ali y Taghavi, Allahvirdi (2007). The impacts of acquiring ISO 9000 certification on the performance of SMEs in Tehran. The TQM Magazine, Vol. 19, No. 2, pp. 140-149.

Beltrán, Jaime (2002). El control de los dispositivos de seguimiento y medición en la norma EN 9100. Forum de Calidad, Vol. 14, No 135, pp. 66-69.

Benner, Mary y Veloso, Francisco (2008). ISO 9000 practices and financial performance: a technology coherence perspective. Journal of Operations Management, Vol. 26, No. 5, pp. 611629.

Bhuiyan, Nadia y Alam, Nadeem (2005). An investigation into issues related to the latest version of ISO 9000. Total Quality Management and Business Excellence, Vol. 16, No. 2, pp. 199-213.
Boiral, Olivier (2011). Managing with ISO systems; lesson from practice. Long Range Planning, Vol. 44, No. 3, pp. 197-220.

Boiral, Olivier y Amara, Nabil (2009). Paradoxes of ISO 9000 performance: a configurational approach. Quality Management Journal, Vol. 16, No. 3, pp. 36-60.

Braun, Boris (2005). Building global institutions: the diffusion of management standards in the world economy, an institutional perspective. In Alvstam, Claes y Schamp, Elke (eds), Linking Industries across the world. London: Ashgate, pp. 3-27.

Brown, Alan; Van del Wiele, Tom y Loughton, Kate (1998). Smaller enterprises' experiences with ISO 9000. International Journal of Quality \& Reliability Management, Vol. 15, No. 3, pp. 273-285

Buttle, Francis (1997). ISO 9000: marketing motivations and benefits. International Journal of Quality \& Reliability Management, Vol. 14, No. 9, pp. 936-947.

Calisir, Fethi (2007). Factors affecting service companies' satisfaction with ISO 9000. Managing Service Quality, Vol. 17, No. 5, pp. 579-593.

Camisón, Cesar; Boronat, Montserrat; Villar, Ana y Puig, Alba (2009). Sistemas de gestión de la calidad y desempeño: importancia de las prácticas de gestión del conocimiento y de I+D. Revista Europea de Dirección y Economía de la Empresa, Vol. 18, No. 1, pp. 123-134.

Casadesus, Martí; Heras, Iñaki y Ochoa, Carlos (2000). Implantación de la normativa ISO 9000 en las comunidades autónomas de Cataluña y el país vasco: conclusiones de dos estudios empíricos. Revista de Dirección y Admi- 
nistración de Empresas, Vol. 8, Septiembre, pp. 43-60.

Casadesus, Martí y Giménez, Gerusa (2000). The benefits of implementation of the ISO 9000 standard: empirical research in 288 spanish companies. TQM Magazine, Vol. 12, No. 6, pp. 432-441.

Casadesus, Martí y Heras, Iñaki (2001). La Norma ISO 9000: beneficios de su introducción en las empresas españolas. Un estudio empírico. Revista Europea de Dirección y Economía de la Empresa, Vol. 10, No. 1, pp. 55-67.

Casadesus, Martí; Karapetrovic, Stanislav y Heras, Iñaki (2004). Beneficios y costes de la implantación de la normativa de calidad ISO 9000: Un estudio comparativo (1998-2002). Revista de Economía y Empresa, Vol. 21, No. 51, pp. 57-74.

Climent, Salvador (2005). Los costes, gastos, burocracia e incremento de productividad por la certificación en la norma ISO 9000 en las empresas certificadas en la norma ISO 9000 de la Comunidad Valenciana. Investigaciones Europeas de Dirección y Economía de la Empresa, Vol. 11, No. 1, pp. 245-259.

Corbett, Charles; Montes, María y Kirsch, David (2005). The Financial Impact of ISO 9000 Certification in the United States: An Empirical Analysis. Management Science, Vol. 51, No. 7, pp. 1046-1059.

Criado, Fernando y Calvo, Arturo (2009). Factores clave y rendimiento en empresas españolas con sistemas de gestión de la calidad. Revista Europea de Dirección y Economía de la Empresa, Vol. 18, No. 2, pp. 107-126.

DBK (2012). Industria Aeroespacial. Resumen informe sectorial elaborado en Sept-2012, disponible en http://www.dbk.es/pdf/sectores/su-
marios/Industria\%20Aeroespacial.pdf.

Dick, Gavin; Heras, Iñaki y Casadesús, Martí (2008). Shedding light on causation between ISO 9001 and improve business performance. International Journal of Operations and Production Management, Vol. 28, No. 7, pp. 687-708.

Douglas, Alex; Coleman, Shirley y Oddy, Richard (2003). The Case for ISO 9000. The TQM Magazine, Vol. 15, No. 5, pp. 316-324.

Escanciano, Carmen; Fernández, Esteban y Vázquez, Camilo (2001). ISO 9000 certification and quality management in Spain: results of a national survey. TQM Magazine, Vol. 13, No. 3, pp. 192-200.

Feng., Mei; Terziovski, Mile y Samson, Danny (2008). Relationship of ISO 9001:2000 quality certification with operational and business performance: a survey in Australia and New Zealand-based manufacturing and service companies. Journal of Manufacturing Technology Management, Vol. 19, No. 1, pp. 22-37.

Gavín, Dick (2000). ISO 9000 certification benefits, reality or myth?. TQM Magazine, Vol. 12, No. 6, pp. 365-371.

Guler, Isin; Guillén, Mauro y MacPherson, John (2002). Global competition, institutions, and the diffusion of organizational practices: the international spread of ISO 9000 quality certificates. Administrative Science Quarterly, Vol. 47, No. 2, pp. 207-232.

Gutiérrez, Evaristo (2008). UNE-EN 9100:2003. Gestión de la calidad en el sector aeronáutico, Forum Calidad. Vol. 1, No. 97/08, pp. 27-30.

Grijalbo, Mercedes y Prida, Bernardo (2005a). La implantación de las normas EN 9100 y el esquema de certificación -Other Party- en España. Revista 
Dyna Ingeniería e Industria, Vol. 80, No. 8, pp. 37-41.

Grijalbo, Mercedes y Prida, Bernardo (2005b). Aviones con certificado de calidad (I): la implantación de la Norma EN 9100. Qualitas hodie: Excelencia, desarrollo sostenible e innovación, Vol. 108, pp. 55-58.

Grijalbo, Mercedes y Prida, Bernardo (2005c). Aviones con certificado de calidad (y II). Qualitas hodie: Excelencia, desarrollo sostenible e innovación, Vol. 109, pp. 32-34.

Hareton, Leung; Keith, Chan y Lee, T. (1999). Costs and benefits of ISO 9000 series: a practical study. International Journal of Quality \& Reliability Management, Vol. 16, No. 7, pp. 675-691.

Hendricks, Kevin y Singhal, Vinod (1996). Quality awards and the market value of the firm: an empirical investigation. Management Science, Vol. 42, No. 3, pp. 415-436.

Heras, Iñaki y Boiral, Olivier (2013). ISO 9001 and ISO 14001: Towards a research agenda on management system standards. International Journal of Management Review, Vol. 15, No. 1, pp. 47-65.

Hopsort, Laurent y Frances, Michel (2011). EN 9100: decryptage dans un contexte aeronautique. Francia: Afnor Editions.

IAQG (2009). The EN 9100 Standard. Inglaterra: International Aerospace Quality Group Editions.

Jang, Woan y Lin, Ching (2008). An integrated framework for ISO 9000 motivation, depth of ISO 9000 implementation and firm performance: the case of Taiwan. Journal of Manufacturing Technology Management, Vol. 19, No. 2, pp. 194-216.

Juny, Jorge (2005). Calidad en el sector aeronáutico: UNE-EN 9100 una norma muy interesante, Forum de Calidad, Vol. 17, No. 161, pp. 39-43.
Lee, Peter; To, W.M. y Billy, T.W. (2009). The implementation and performance outcomes of ISO 9000 in service organizations: an empirical taxonomy. International Journal of Quality and Reliability Management, Vol. 26, No. 7, pp. 646-662.

Lo, Chris; Yeung, Andy y Cheng, T.C. (2009). ISO 9000 and supply chain efficiency: Empirical evidence on inventory and account receivable days. International Journal of Production Economics, Vol. 118, No. 2, pp. 367-374.

Lo, Liang y Chang, Dong (2007). The difference in the perceived benefits between firms that maintain ISO certification and those that do not. International Journal of Production Research, Vol. 48, No. 5, pp. 1881-1897

Mann, Robin y Kehoe, Dennis (1994). An evaluation of the effects of quality improvement activities on business performance. International Journal of Quality \& Reliability Management, Vol. 11, No. 4, pp. 29-44.

Martínez-Costa, Micaela y Martínez-Lorente, Angel (2007). A triple analysis of ISO 9000 effects on company performance. International Journal of Productivity and Performance Management, Vol. 56, No. 5/6, pp. 484-499.

Martínez-Costa, Micaela; Martínez-Lorente, Angel y Choi, Thomas (2008). Simultaneous consideration of TQM and ISO 9000 on performance and motivation: An empirical study of Spanish companies. International Journal of Production Economics, Vol. 113, No. 1, pp. 23-39.

Martinez-Costa, Micaela; Choi, Thomas; Martínez, Jose y Martinez-Lorente Angel (2009). ISO 9000/1994, ISO 9001/2000 and TQM: The performance debate revisited. Journal of Operations Management, Vol. 27, No. 6, pp. 495-511. 
Mercado, Carmelo; Castillo, Carlos y Mateo, Antonio (2005). La Normativa ISO 9000 como herramienta para incrementar el valor percibido por el cliente en las entidades financieras españolas, en González, Federico y Terceño, Antonio (ed.) "Simultaneidad Decisional y Multifocalidad Empresarial”. España. AEDEM Ediciones, pp. 350-367.

Mugarra, Pedro (2003). Experiencia de SENER respecto a la Norma EN 9100. UNE-Boletín mensual AENOR, Vol. 176, pp. 36-40.

Murga, Jesús (2002). Experiencia de ITP en Calidad. Revista Dyna de Ingeniería e Industria, Vol. 77. No. 7, pp. 32-36.

Nicolau, Juan Luis y Sellers, Ricardo (2003). Influencia de la calidad sobre la rentabilidad y la volatilidad. Revista de Economía Aplicada, Vol. 11, No. 33, pp. $65-82$.

Psomas, Evangelos; Fotopoulos, Christos y Kafetzopoulos, Dimitrius (2010). Critical Factors for Effective Implementation of ISO 9001 in SME Service Companies. Managing Service Quality, Vol. 20, No. 5, pp. 440-457.

PRICEWATERHOUSECOOPERS (PwC) (2012). Flight International Aerospace Top 100. Disponible en http://www.pwc.com/gx/en/aerospace-defence/publications/top-performing-aerospace-companies-forgrowth.jhtml.

Rodriguez, Javier; Gonzalez, Javier y Martínez, Angel (2006). An analysis of the degree of small companies' dissatisfaction with ISO 9000 certification. Total Quality Management, Vol. 17, No. 4, pp. 507-521.

Romano, Pietro (2000). ISO 9000: What is its impact on performance?. Quality Management Journal, Vol. 7, No. 3, pp. 38-56.
Sampaio, Paulo; Saraiva, Pedro y Rodrigues, Antonio (2009). ISO 9001 certification research: questions, answers and approaches. International Journal of Quality and Reliability Management, Vol. 26, No.1, pp. 38-58.

Santos, Lucía y Escanciano, Carmen (2002). Análisis de la influencia de la certificación ISO 9000 sobre los resultados empresariales: implicaciones en el ámbito de la calidad total y de la orientación al mercado. Revista ICADE, Vol. 55, pp. 1-30.

Sharma, Divesh (2005). The association between ISO 9000 certification and financial performance. International Journal of Accounting, Vol. 40, No. 2, pp. 151-172.

Singels, Jeroen; Rúel, Gwenny y Van der Water, Henny (2001). ISO 9000 series. Certification and performance. International Journal of Quality and Reliability Management, Vol. 18, No. 1, pp. 62-75.

Taddei, Daniel (2007). Le management en suivant la norme EN 9100. Francia: Societé des Ecrivains.

Tari, Juan; Molina-Azorín, Jose y Heras, Iñaki (2012). Benefits of ISO 9001 and ISO 14001 standards: a literature review. Journal of Industrial Engineering Management, Vol. 5, No. 2, pp. 297332.

Terlaak, Ann y King, Andrew (2006). The effect of certification with the ISO 9000 Quality Management Standard: A signalling approach. Journal of Economics Behavior \& Organization, Vol. 60 , No. 4 , pp. 579-602.

Terziovski, Mile y Power, Damien (2007). Increasing ISO 9000 certification benefits: a continuous improvement approach. International Journal of Quality and Reliability Management, Vol. 24, No. 2, pp. 141-163. 
Terziovski, Mile; Samson, Danny y Dow, Douglas (1997). The business value of quality management systems certification. Evidence from Australia and New Zealand. Journal of Operations Management, Vol. 15, No. 1, pp. 1-18.

Tsiotras, George y Gotzamani, Katerina (1996). ISO 9000 as an entry key to TQM: the case of Greek Industry. International Journal of Quality and Reliability Management, Vol. 13, No. 4, pp. 64-76.

Vilar, José (2003). La implantación de la norma EN 9100 en EADS-CASA. UNE-Boletín mensual de AENOR, Vol. 177, pp. 42-45.

Vinuesa, Luz María (2007). La influencia de la certificación ISO 9000 sobre los resultados. Revista Iberoamericana de Contabilidad de Gestión, Vol. 10, pp. 145-176.

Vloeberghs, Daniel y Bellens, Jan (1996). Implementing the ISO 9000 standards in
Belgium. Quality Progress, Vol. 29, No. 6, pp. 43-48.

Wahid, Roslina y Corner, James (2009). Critical success factors and improvements in ISO 9000 maintenance. International Journal of Quality \& Reliability Management, Vol. 26, No. 9, pp. 881-893.

Wayhan, Victor; Kirche, Elias y Khumawala, Basheer (2002). ISO 9000 certification: The financial performance implications. Total Quality Management, Vol. 13, No. 2, pp. 217-231.

Wensveen, John (2011). Air Transportation: a management perspective. $7^{\text {th }}$ Edition. Ashgate Publishing Company. Burlington (USA).

Zaramdini, Walid (2007). An empirical study of the motives and benefits of ISO 9000 certification: The UAE experience. International Journal of Quality and Reliability Management, Vol. 24, No. 5, pp. 472-491. 